\title{
Hierro, oxígeno y desarrollo placentario en la génesis de la preeclampsia. Efectos de la altura en Ecuador
}

\author{
Carlos Escudero ${ }^{1}$, Andrés Calle $2 \mathrm{a}$. \\ Iron, oxygen and placental \\ development in the etiology \\ of preeclampsia. \\ Effects of high altitude in Ecuador
}

Preeclampsia is the second cause of maternal death in Ecuador. The etiology of this condition is probably a placental alteration, although the details are not well known. The development of the placenta is closely related to the availability of oxygen. A defect in the differentiation of trophoblastic cells due to a faulty sensitization to changes in oxygen pressure, could be the cause of the alteration in placental development. The role of iron and local environmental conditions of a susceptible population, should be considered in the study of the etiology of preeclampsia. In the Andrean area of Ecuador, the high incidence of preeclampsia could be explained by the high prevalence of anemia and high altitude. However more studies are required to establish a close link between the environmental conditions of this area and the imperfect placental development (Rev Méd Chile 2006; 134: 491-8).

(Key words: Anemia, iron-deficiency; Placenta; pre-eclampsia)

Recibido el 28 de octubre, 2004. Aceptado el 30 de septiembre, 2005.

${ }^{1}$ Programa de Doctorado en Ciencias Médicas, Pontificia Universidad Católica de Chile. ${ }^{2}$ Centro de Biomedicina, Universidad Central del Ecuador.

a MsC

La a preeclampsia es una enfermedad sistémica caracterizada por hipertensión, proteinuria y coagulopatía, que se manifiesta a partir del segundo trimestre del embarazo ${ }^{1}$. La epidemiología de esta enfermedad muestra diferencias muy marcadas en relación al sitio de estudio. En Ecuador

Correspondencia a: Dr. Carlos Escudero. Programa de Doctorado en Ciencias Médicas, Pontificia Universidad Católica de Chile. Phone 56- 2-3548120. E mail: cescuder@med.puc.cl alcanza cifras tan altas como $12 \%$, como recientemente reportamos ${ }^{2}$. Además, complica al recién nacido, principalmente por retardo de crecimiento y parto de pretérmino iatrogénico ${ }^{3}$. Pese a ello, la etiopatogenia de esta enfermedad no ha sido determinada. Sin embargo, se reconocen dos acontecimientos patológicos íntimamente relacionados. Así, una inicial alteración en el proceso de placentación lleva a la formación de una placenta hipóxica y disfuncional, capaz de liberar hacia la circulación materna factores» que causan disfun- 
ción del endotelio vasculart-8. Los mecanismos moleculares de la interacción entre ambos procesos patológicos han documentado la importancia de los cambios en la presión de oxígeno $\left(\mathrm{PO}_{2}\right)$, de las moléculas involucradas en reconocer estos cambios y de la susceptibilidad materna a los mismos ${ }^{9-13}$.

En el presente artículo revisaremos los mecanismos identificados de diferenciación celular y desarrollo placentario. Además, señalaremos condiciones particulares en la sierra ecuatoriana en donde se podrían sumar varios agravantes, como la altura y la deficiencia de hierro, que determinan una mayor susceptibilidad de preeclampsia en esta población.

\section{EL HIERRO Y EL OXÍGENO \\ EN EL DESARROLLO PLACENTARIO NORMAL}

La placenta es un órgano transitorio que forma la interfase física y funcional entre la madre y el embrión en desarrollo. Durante el proceso de formación de la placenta, se reconocen varias líneas celulares. El trofoblasto o células madre, genera al sincitiotrofoblasto y al trofoblasto invasivo. El sincitiotrofoblasto es el responsable del mantenimiento de nutrientes placentarios, intercambio gaseoso, producción hormonal y factores de crecimiento. El trofoblasto invasivo puede ser diferenciado en intersticial y endovascular. Ambos invaden los vasos sanguíneos maternos, y reemplazan los componentes de los mismos, transformándolos en vasos de capacitancia ${ }^{14}$.

Durante las primeras semanas de gestación, el desarrollo placentario se realiza en un medio hipóxico. Así, estudios por ecografía Doppler han demostrado que el flujo sanguíneo en la placenta, aparece al final del primer trimestre de gestación ${ }^{15,16}$. Además, al cuantificar directamente la $\mathrm{PO}_{2}$ se demostró que a las 8 semanas de gestación, ésta fue menor a $15 \mathrm{mmHg}$ e incrementó a más de $50 \mathrm{mmHg}$ a las 12 semanas $^{16}$.

Este cambio en la $\mathrm{PO}_{2}$, es muy importante para el desarrollo placentario. Trabajos iniciales ${ }^{9,17,18}$ muestran que a $\mathrm{PO}_{2}$ bajas, comparables a las que existen durante el embarazo temprano, se estimula la proliferación del trofoblasto e inhiben la diferenciación de éste hacia el fenotipo invasivo. Además, la utilización de quelantes de hierro, como la deferroxamina (DFO), induce crecimien- to del tejido placentario ${ }^{18}$. La DFO ha sido utilizada en modelos de hipoxia, debido a su capacidad para inducir actividad del factor inducido por hipoxia tipo 1 alfa (HIF $1 \alpha$ ), un conocido factor de transcripción, que media el incremento en la expresión de moléculas involucradas en la adaptación a la hipoxia como eritropoyetina, factor de crecimiento endotelial, calicreína (Escudero $\mathrm{C}$, datos no publicados) y varias enzimas involucradas en la generación de energía en condiciones hipóxicas ${ }^{19}$.

El HIF 1 está constituido por dos subunidades, la alfa $(\alpha)$ y la beta $(ß)$. El HIF-1ß, es constitutivo, mientras que el HIF- $1 \alpha$ es modulable en forma de oxígeno dependiente. Así, en condiciones de normoxia, es hidroxilado en los residuos de prolina 564 y 402, vía prolil-hidroxilasas. Posteriormente es reconocido por la proteína de von Hippel-Lindau (VHL), la cual a su vez es señal para formar un complejo de ubiquitilación, que es indicativa de degradación por proteosomas ${ }^{19,20}$. Adicionalmente, se reconoce una tercera hidroxilación en el residuo aspargina 803, que impide su acoplamiento a proteínas nucleares involucradas en la transcripción de genes regulados por HIF ${ }^{21}$. Sin embargo, en condiciones de hipoxia o en presencia de quelantes de hierro, las hidroxilasas dejan de modificar al HIF-1 $\alpha$ y éste se acopla al HIF-1ß para ser traslocado al núcleo donde funciona como factor de transcripción ${ }^{19-22}$.

Al momento existen varias publicaciones demostrando una incrementada concentración de HIF durante el proceso de placentación normal y patológica ${ }^{17,18}$. Así, se ha sugerido que el HIF, durante la fase hipóxica del desarrollo placentario, activa factores de crecimiento que inhiben la diferenciación del trofoblasto, como el factor de crecimiento transformante tipo 3 (18, TGF- $乃$-3) y que por algún motivo, todavía no determinado, esta inhibición se mantendría en la preeclamp$\operatorname{sia}^{23,24}$.

En este sentido, merece realizar algunas puntualizaciones en esta vía de percepción de oxígeno, debido a la trascendencia de las observaciones. El sensor de oxígeno ha sido relacionado con la disponibilidad de hierro. Así, inicialmente, se consideró que podría ser una hemoproteína (proteína asociada a grupo HEM) que estaría localizada a nivel citoplasmático y, eventualmente, en la mitocondria $^{9,10}$. Interesantemente, el descubrimiento 
del HIF y de sus mecanismos de regulación, han demostrado que las prolil-hidroxilasas, son dependiente de hierro aunque no de grupos HEM ${ }^{11}$. Así, el hierro podría constituirse en un elemento limitante dentro del sistema sensor de oxígeno.

Durante el desarrollo placentario, el rol del hierro no ha sido estudiado. Sin embargo, existen interesantes evidencias clínicas que soportan la dependencia del mismo en este delicado proceso. Así, durante el embarazo temprano, la concentración de hemoglobina, hierro y ferritina se correlacionan inversamente con la cantidad de hormonas placentarias circulantes ${ }^{25}$. Además, el hierro pudiera incluso determinar el volumen placentario 26 o una mayor susceptibilidad a apoptosis en células placentarias ${ }^{27}$.

Por otro lado, la utilización de oxígeno lleva a la formación, entre otras cosas, de radicales libres $(\mathrm{ROS})^{12}$. En este sentido, se conoce que el hierro participa en la formación de ROS, mediante la conocida reacción de Fenton ${ }^{30,31}$. En condiciones de hipoxia, como las que suceden en la primera etapa de diferenciación trofoblástica, existe una sobreproducción de ROS que modula la diferenciación del mismo ${ }^{28}$, en donde la participación del hierro podría estar involucrada e, inclusive, modular la actividad de HIF y a la expresión de genes dependientes de cambios oxidativos ${ }^{12,28,29}$.

\section{EL DESARROLLO PLACENTARIO ALTERADO \\ EN LA PREECLAMPSIA: CONSECUENCIA DE FACTORES MATERNOS Y MEDIO AMBIENTALES}

Al momento existen varias evidencias que señalan a la preeclampsia como una enfermedad que cursa con una alteración en el desarrollo placentario (Tabla 1). Así, el trofoblasto en la preeclampsia

Tabla 1. Evidencias de la alteración en la función placentaria durante la preeclampsia

\begin{tabular}{|ll|}
\hline Evidencias & Patología asociada \\
\hline Observacionales & Presencia de preeclampsia en embarazos donde no hay feto, \\
& mola hidatiforme \\
& Incremento del volumen placentario \\
& Mayor tasa de preeclampsia en patologías que cursan con \\
& incremento del volumen placentario (gemelar, diabetes) \\
Morfológicas & Endoteliosis placentaria \\
& Depósitos grasos, similares a ateromas \\
& Conservación de características arteriales de vasos maternos \\
Bioquímicas & Incrementada producción de ROS* \\
& Disminuida actividad de citocromo C oxidasa en mitocondrias placentarias \\
& Incremento del activador de plasminógeno tipo 2 \\
& Disminución de antioxidantes en tejido placentario \\
& Alteración en la producción de óxido nítrico (NO) \\
Funcionales & Incremento de la respuesta a angiotensina II de vasos desiduales \\
& Alteración en la invasión trofoblástica \\
& Alteración en la diferenciación trofoblástica \\
& Disminución del número de vasos maternos invadidos \\
& Alteración en la producción de metaloproteinasas \\
& Reacciones inmunológicas contra trofoblasto \\
& Incremento de apoptosis en células placentarias \\
\end{tabular}

*ROS; especies reactivas de oxígeno 
mantiene un fenotipo proliferativo, que produce un mayor crecimiento placentario y que, además, su diferenciación hacia un fenotipo invasivo está alterada, determinando que los vasos sanguíneos maternos conserven sus propiedades de contracción, llevando, en último término, a que las condiciones de hipoxia sigan presentes inclusive en el tercer trimestre de gestación ${ }^{32}$. Los mecanismos moleculares de estas alteraciones aún no son bien entendidos, sin embargo, recientemente se ha demostrado una sobreexpresión y actividad de HIF-123,24, TGF $33^{17}$ y de ROS ${ }^{33}$ en explantes de placentas de mujeres con preeclampsia, sugerentes de la conservación de la señal de hipoxia.

En este sentido, un modelo en humanos, que asemeja en mucho los cambios debido a hipoxia en la placenta, es la vida en la altura. Así, se conoce que en la altura, el desarrollo placentario presenta características adaptativas muy particulares y que la presentación de preeclampsia es 2 a 4 veces más frecuente ${ }^{34,35}$. Los eventos moleculares de este modelo han sido analizados recientemente. Así, se ha demostrado que durante la preeclampsia existe una activación en la transcripción genética similar a la evidenciada en la altura o en condiciones de hipoxia ${ }^{36}$. Interesantemente, este comportamiento ha sido relacionado con la conservación de un fenotipo proliferativo en las células placentarias ${ }^{36}$.

Por otro lado, se conoce también que vivir en la altura conlleva a un incrementado estado de generación de ROS y de consumo de antioxidantes ${ }^{40}$. En el campo de la preeclampsia, existen varios trabajos, incluidos los nuestros ${ }^{41-45}$, en los que se señala una incrementada generación de ROS y consumo de antioxidantes en muestras de mujeres con preeclampsia. En este sentido, si bien se conoce la asociación, la explicación de la(s) vía(s) molecular(es) aún no han sido determinadas y, probablemente, el solo hecho de vivir en la altura tampoco explicaría completamente la génesis de la preeclampsia. Es por ello que, se considera que la preeclampsia, como entidad nosológica, debe mostrar características particulares dependientes del medio ${ }^{37}$. Así, probablemente, mecanismos como la homeostasis del hierro, o de oxidantes-antioxidantes $\mathrm{y}$, en definitiva, la forma de cómo el trofoblasto percibe las concentraciones de oxígeno sean hitos claves en el proceso de entendimiento de esta alterada res- puesta en poblaciones susceptibles como la residente en la altura.

Al momento, existen interesantes aproximaciones de tipo clínicas. Perry y cols ${ }^{26}$, en un estudio prospectivo, en el que se cuantificó la concentración de hemoglobina, un marcador grueso de la concentración de hierro, a las 16 semanas de gestación, evidenció que aquellas mujeres con valores bajos de hemoglobina mostraban una tendencia al incremento en el volumen placentario al final del mismo, aunque esta relación no fue estadísticamente significativa. Más recientemente, Kadvrov y cols ${ }^{27}$ mostraron que las mujeres con anemia presentaban una incrementada apoptosis en el trofoblasto. Adicionalmente, estudios genómicos en familias con historia de preeclampsia $^{38,39}$ han mostrado que alteraciones en moléculas dependientes de hierro como el citocromo c oxidasa (complejo IV mitocondrial) determinan una mayor susceptibilidad para el desarrollo de la patología.

Con estas evidencias, la forma de percibir los cambios de oxígeno en las primeras etapas de desarrollo placentario podrían determinar el normal o anormal desarrollo de la misma, consecuentemente predisponer al desarrollo de preeclampsia. De ser así, condiciones como la altura (hipoxia), la anemia (bajo hierro) 0 el desequilibrio entre oxidantes-antioxidantes en las primeras etapas de gestación mantendrían la cascada se señalización hipóxica inclusive hasta el término de la gestación.

\section{CONSIDERACIONES DEL EMBARAZO PATOLÓGICO EN ECUADOR}

Durante varios años, hemos estudiado la respuesta materna en el embarazo complicado con preeclampsia 2,42,43,46-49, encontrando una secuencia de acontecimientos que tienen que ver con la alteración del endotelio materno y en la producción de óxido nítrico (NO). Descubrimientos corroborados por otros grupos, que inclusive, han documentado la utilidad de antioxidantes en la prevención de preeclampsia 44,50 . Este mismo estudio, nos obliga a buscar respuestas en los procesos de placentación y sus alteraciones en esta enfermedad.

Así, en Ecuador, la anemia gestacional por deficiencia de hierro alcanza cifras tan altas como 
$26 \% 51$. Es por ello que iniciamos estudios clínicos para revertir el efecto ${ }^{52,53}$ y ahora es un problema del que se está ocupando el Ministerio de Salud Pública. Sin embargo, esta estrategia, si bien ha reducido los índices de mortalidad materna, no ha conseguido los objetivos deseados. Y es que, de acuerdo a nuestra observación, la medida puede estar empezando demasiado tarde ${ }^{53}$. Más aún, la suplementación con hierro durante la etapa final del embarazo, cuando el cuadro de preeclampsia está instaurado, pudiera incluso agravar el mismo debido a una mayor generación de $\mathrm{ROS}^{54}$. Según este paradigma, la suplementación de hierro debería involucrar a mujeres en edad fértil y combatir la deficiencia subclínica ${ }^{54}$ para que no sólo se mejore la tasa de mortalidad total, sino también la de preeclampsia.

Otra observación importante en Ecuador, es el hecho de que existe una diferenciación muy marcada entre las tasas de preeclampsia de las regiones de la sierra y de la costa. Así, en la sierra (Quito $2.860 \mathrm{~m}$ de altura) encontramos una tasa de preeclampsia de $12-15 \%$, mientras que en la costa (Guayaquil, a nivel del mar) es de 5 a $7 \% 55$. $\mathrm{Si}$ bien, esto puede deberse a diferencias propias de cada población, en el marco de la PO2, y la cascada de señalización dependiente de ésta, pudiera también deberse a características como la dieta o la altura. Así, las poblaciones en la región de la sierra están constitucionalmente en un estado de consumo de antioxidantes e hipoxia crónica, que influiría en la alta presentación de preeclampsia ${ }^{56}$.

\section{REFERENCIAS}

1. National High Blood Pressure Education Working Group Report on High Blood Pressure in Pregnancy. Am J Obstet Gynecol 1990; 163: 1689-712.

2. Terán E, Escudero C, CaLe A. C reactive protein during normal pregnancy and preeclampsia. Int $\mathrm{J}$ Gynaecol Obstet 2005; 89: 299-300.

3. Zupan J. Perinatal mortality in developing countries. N Engl J Med 2005; 352: 2047-8.

\section{CONCLUSIONES}

Los estudios experimentales han demostrado la necesidad de los cambios en la $\mathrm{PO}_{2}$ en el medio trofoblástico, para una adecuada diferenciación celular e implantación placentaria durante el embarazo normal. En la preeclampsia, este proceso estaría alterado.

Si bien, la preeclampsia ha sido considerada como la enfermedad de las teonías, es también cierto que el papel matemo y medio ambiental no puede ser sacado de escena en la presentación del mismo. En este sentido, las deficiencias nutricionales durante las primeras etapas del embarazo e inclusive antes del mismo, pueden explicar, al menos en parte, el alto porcentaje de esta patología en poblaciones susceptibles $^{2,56}$. El rol del hiemo y de los antioxidantes deberá ser incluido dentro del estudio de la patogénesis de la preeclampsia, con la salvedad de abarcarlos en etapas tempranas del embarazo o inclusive antes del mismo, como lo recomiendan observadores de la Organización Mundial de la Salud ${ }^{57,58}$.

Si bien reconocemos que los datos revisados son una asociación muy gruesa sobre mecanismos moleculares específicos, es también motivo de interesantes hipótesis que podrían guiar al estudio de susceptibilidad a la enfermedad en poblaciones específicas. Es por ello que son necesarios estudios a nivel molecular dirigidos a determinar las interacciones entre ROS y antioxidantes, hierro, prolil- hidroxilasas, considerando siempre el margen de semanas en donde ocurren los cambios más importantes como la diferenciación y conversión del trofoblasto.

4. Redman CW, SARgent IL. Latest advances in understanding preeclampsia. Science 2005; 308: 1592-4.

5. Milne F, Redman C, Walker J, Baker P, Bradiey J, COOPER C ET AL. The pre-eclampsia community guideline (PRECOG): how to screen for and detect onset of pre-eclampsia in the community. BMJ 2005; 330: 576-80.

6. Sibai B, DekKer G, Kupferminc M. Pre-eclampsia. Lancet 2005; 365: 785-99. 
7. GoLdman-Wohl D, Yagel S. Regulation of trophoblasto invasion: from normal implantation to preeclampsia. Mol Cell Endocrinol 2002; 187: 233-8.

8. ReDMan C. Current topic: pre-eclampsia and the placenta. Placenta 1991; 12: 301-8.

9. Genbacev O, Zhou Y, Ludlow J, Fisher S. Regulation of human placental development by oxygen tension. Science 1997; 277: 1669-72.

10. De Marco C, Caniggia I. Mechanisms of oxygen sensing in human trophoblast cells. Placenta 2002; 23: S58-S68.

11. SEMENZA GL Hydroxylation of HIF-1: oxygen sensing at the molecular level. Physiology (Bethesda) 2004; 19: 176-82.

12. Brunele JK, Bell EL, Quesada NM, Vercauteren K, Tiranti V, Zeviani M et al. Oxygen sensing requires mitochondrial ROS but not oxidative phosphorylation. Cell Metab 2005; 1: 409-14.

13. SEMENZA GL. O2-regulated gene expression: transcriptional control of cardiorespiratory physiology by HIF-1. J Appl Physiol 2004; 96: 1173-7.

14. LYAL F. Priming and remodelling of human placental bed spiral arteries during pregnancy-a review. Placenta 2005; 26: S31-6.

15. JAFFE R, WARSON S. Transvaginal color Doppler imaging in the assessment of uteroplacental blood flow in the normal first trimester pregnancy. Am J Obstet Gynecol 1991; 164: 781-5.

16. Jauniaux E, Watson A, Hempstock J, Bao Y, Skepper J, BURTON G. Onset of maternal arterial blood flow and placental oxidative stress. A possible factor in human early pregnancy failure. Am J Pathol 2000; 157: 2111-22.

17. Genbacev O, Josun R, Damsky C, Рошоті B, Ficher $\mathrm{S}$. Hypoxia alters early gestation human cytotrophoblast differentiation/invasión in vitro and models the placental defects that occur in preeclampsia. J Clin Invest 1996; 97: 540-50.

18. Caniggia I, Mostachfi $\mathrm{H}$, Winter J, Gassmann M, Lye S, Kuliszewsi M et al. Hypoxia-inducible factor -1 mediates the biological effects of oxygen on human trophoblast differentiation through TGFß3. J Clin Invest 2000; 105: 577-87.

19. SaFran M, Kaelin WG JR. HIF hydroxylation and the mammalian oxygen-sensing pathway. J Clin Invest 2003; 111: 779-83.

20. JaAkkola P, Mole DR, Tian YM, Wilson MI, Gielbert J, GASKELL SJ ET AL. Tangeting of HIF-alpha to the von Hippel-Lindau ubiquitylation complex by
O2-regulated prolyl hydroxylation. Science 2001; 292: 468-72.

21. Lando D, Peet DJ, Whelan DA, Gorman JJ, WhiteLAW ML Asparagine hydroxylation of the HIF transactivation domain a hypoxic switch. Science 2002; 295: 858-61.

22. Emerung BM, Piatanias LC, Black E, Nebreda AR, Davis RJ, Chandel NS. Mitochondrial Reactive Oxygen Species Activation of p38 Mitogen-Activated Protein Kinase Is Required for Hypoxia Signaling. Mol Cell Biol 2005; 25: 4853-62.

23. Rajakumar A, Brandon HM, Daftary A, Ness R, CONRAD KP. Evidence for the functional activity of hypoxia-inducible transcription factors overexpressed in preeclamptic placentae. Placenta 2004; 25: 763-9.

24. Rajakumar A, Doty K, Daftary A, Markovic N, CONRAD KP. Expression of von Hippel Lindau (pVHL) Protein in Placentae from Normal Pregnant Women and Women with Preeclampsia. Placenta 2006; 27: 411-21.

25. Wheeler T, Solerero C, Aiderman S, Landen J, ANTHONy F, Osmond C. Relation between maternal haemoglobin and placental hormone concentration in early pregnancy. Lancet 1994; 343: 511-13.

26. Perry I, Beevers B, Whincup P, Bareford D. Predictors of ratio of placental weight to fetal weight in multiethnic community. BMJ 1995; 310: 436-39.

27. Kadyrov M, Schmitz C, Black S, Kaufmann P, HuPPERTZ B. Pre-eclampsia and maternal anaemia display reduced apoptosis and opposite invasive phenotypes of extravillous trophoblast. Placenta 2003; 24: 540-8.

28. FreEman M, CRAPo J. Hypoxia increases oxygen radical production in rat lungs and lung mitochondria. J Biol Chem 1981; 256: 10986-92.

29. Watson AL, Skepper JN, Jauniaux E, Burton GJ. Susceptibility of human placental syncytiotrophoblastic mitochondria to oxygen-mediated damage in relation to gestational age. J Clin Endocrinol Metab 1998; 83: 1697-705.

30. Duesterberg CK, Cooper WJ, Waite TD. Fentonmediated oxidation in the presence and absence of oxygen. Environ Sci Technol 2005; 39: 5052-8.

31. Dendorfer A, Heidbreder M, Hellwig-Burgel T, Johren O, QADri F, DominIaK P. Deferoxamine induces prolonged cardiac preconditioning via accumulation of oxygen radicals. Free Radic Biol Med 2005; 38: 117-24. 
32. Red-Horse K, Zhou Y, Genbacev O, Prakobphol A, FOULK R, MCMASTER M ET AL. Trophoblast differentiation during embryo implantation and formation of the maternal-fetal interface. J Clin Invest 2004; 114: 744-54.

33. Vanderlelie J, Venardos K, Cufton VL, Gude NM, Ciarke FM, Perkins AV. Increased biological oxidation and reduced anti-oxidant enzyme activity in pre-eclamptic placentae. Placenta 2005; 26: 53-8.

34. Keyes LE, Armaza JF, Niermeyer S, Vargas E, Young DA, Moore LG. Intrauterine growth restriction, preeclampsia, and intrauterine mortality at high altitude in Bolivia. Pediatr Res 2003; 54: 20-5.

35. Palmer SK, Moore LG, Young D, Cregger B, Berman JC, ZAmudio S. Altered blood pressure course during normal pregnancy and increased preeclampsia at high altitude (3100 meters) in Colorado. Am J Obstet Gynecol 1999; 180: 1161-8.

36. Soleymanlou N, Jurisica I, Nevo O, Ietta F, Zhang X, Zamudio $S$ et aL. Molecular Evidence of Placental Hypoxia in Preeclampsia. J Clin Endocrinol Metab 2005; 90: 4299-308.

37. López-JaramLo P, García RG, López M. Preventing pregnancy-induced hypertension: are there regional differences for this global problem? J Hypertens 2005; 23: 1121-9.

38. Vuorinen K, Remes A, Sormune R, Tapanainen J, HASSEINEN I. Placental mitochondrial DNA and respiratory chain enzymes in the etiology of preeclampsia. Obstet Gynecol 1998; 91: 950-5.

39. Furui T, Karauchi O, Tanaka M, Mizutani S, Ozana $\mathrm{T}$, Tomoda Y. Decrese in cytochrome c oxidase and cytochrome oxidase subunit I messenger RNA levels in preeclamptic pregnancies. Obstet Gynecol 1994; 84: 283-88.

40. AsKew EW. Work at high altitude and oxidative stress: antioxidant nutrients. Toxicology 2002; 180: 107-19.

41. López-Jaramilo P, Terán E, Ringevist A, Moya W, RIVERA J, BerRAzUETA JR. Oxidised low-density lipoproteins and nitric oxide during normal pregnancy and preeclampsia. In: Moncada S, Toda N, Maeda H, Higgs EA, eds. Biology of Nitric Oxide Part 6. Portland Press: London. 1998. pp. 322.

42. Terán E, Racines M, Vivero S, Escudero C, Molina G, Caue A. Preeclampsia is associated with a decrease in plasma coenzyme Q10 levels. Free Rad Biolo Med 2003; 35: 1453-56.
43. Terán E, Escudero C, Vivero S, Moya W, Calle A. Alter release of nitric oxide from nitrosoprotein. Am J Obst Gynecol. 2006; 92: 260-1.

44. Poston L, Raijmakers M, Kelu F. Vitamin E in preeclampsia. Ann NY Acad Sci 2004; 1031: 2428.

45. Kharfi A, Giguere $Y$, De Grandpre $\mathrm{P}$, Moutquin JM, ForeST JC. Human chorionic gonadotropin (hCG) may be a marker of systemic oxidative stress in normotensive and preeclamptic term pregnancies. Clin Biochem 2005; 38: 717-21.

46. Terán E, Escudero C, Vivero S, Cale A, Molina G. Plasma nitric oxide levels during normal pregnancy and preeclampsia. A follow study. Hypertension. 2006; 47: 17.

47. Terán E, Escudero C, Vivero S, Enríquez A, Cale A. Intraplatelet cyclic guanosine-3',5'-monophosphate levels during pregnancy and preeclampsia. Hypertens Pregnancy 2004; 23: 303-8.

48. Terán E, Escudero C, Moya W, Flores M, Valuance P, LóPEZ-JARAMILO P. Elevated C-reactive protein and pro-inflammatory cytoquines in Andean women with preeclampsia. IntJ Gynaecol Obstet 2001; 75: 243-9.

49. Terán E, Escudero C, Calle A. Seroprevalence of antibodies of Chlamydia Pneumoniae, Helicobacter Pylori and Streptolysine $\mathrm{O}$ as a risk factor to develop preeclampsia. Obstetric and Gynecology 2003; 102: 198-9.

50. Chappell L, Seed P, Briley A, Kelly F, Lee R, Hunt B ET AL. Effect of antioxidants on the occurrence of pre-eclampsia in women at increased risk: a randomized trial. Lancet 1999; 354: 810-6.

51. Yepez R, Calle A, Galan P, Estévez E, Dávila M, ESTRELAR R ET AL. Iron status in Ecuadorian pregnant women living at 2,800 $\mathrm{m}$ altitude: relationship with infant iron status. Int J Vitam Nutr Res 1987; 57: 327-32.

52. Yepez R, Estevez E, Galan P, Chaulac M, Dávila M, CALE A ET AL. High altitude anemia: validity of definition criteria. Sante: 1994; 4: 9-13.

53. Cale A. Vitaminas. En: Nutrición durante el Embarazo, Primera Edición. Calle A. Autor-Editor. Publicaciones Médicas, 2000. Quito, Ecuador, 2000: 51-101.

54. Sснош TO. Iron status during pregnancy: setting the stage for mother and infant. Am J Clin Nutr 2005; 81: 1218S-22S. 
55. Instituto Nacional Ecuatoriano de Estadísticas y Censos. INEC, 1997.

56. Weigel M, Narváez M, FÉlux C, López A, LópezJARAMLL P. Prenatal diet, nutrient intake and pregnancy outcome in urban Ecuadorian primiparas. Arch Latinoam Nutr 1991; 41: 21.

57. Viliar J, Say L, Shennan A, Lindherimer M, Duley L, Conde-Agudelo A ET AL. Methodological and technical issues related to the diagnosis, screening, prevention, and treatment of preeclampsia and eclampsia. Int J Gynecol Obstet 2004; 85 : S28-S41.

58. Viliar J, Conde-Agudelo A, Lindheimer M. World health organization systematic review of screening tests for preeclampsia. Obstet Gynecol 2004; 104: 1367-91.

Agradecimientos

A la Unidad de Farmacología Experimental y Metabolismo Celular del Centro de Biomedicina de la Universidad Central del Ecuador. CE, es becario MESESUP por el proyecto «nternacionalización del Programa de Doctorado en Ciencias Médicas». PUC № 404. 